\title{
Flexible Ultrasonic Transducers Using Piezoelectric Fiber Composites with Antisymmetric Interdigital Electrodes
}

\author{
Ching-Chung Yin, Yu-Chien Wu, Yu-Shyan Liu, Shih-Ming Hsu \\ Department of Mechanical Engineering \\ National Chiao Tung University \\ Hsinchu 30010, Taiwan, Republic of China \\ E-mail: ccyin@faculty.nctu.edu.tw
}

\begin{abstract}
A conformal ultrasonic transducer constructed by piezoelectric fiber composites (PFC) with new arrangement of electrodes is numerically and experimentally studied. This device is designed to transmit and receive guided waves of constant wavelength for structural health monitoring application. A periodic finite element approach has been used to demonstrate the electric field induced by symmetric or anti-symmetric electrodes as well as working principle of this device interacted with the host plate. The extensional mode of this device is used to actuate both $A_{0}$ and $S_{0}$ modes of Lamb waves in the host plate. The added mass effect of PFC has more notable influence on transmission of $S_{0}$ modes than $A_{0}$ mode in the area near both edges of PFC. The linear scan of transmitted acoustic waveforms along the axis in front of the transducer by a laser Doppler vibrometer reveals the near-field interference pattern, which is caused by the guided waves of constant wavelength.
\end{abstract}

Keywords-flexible transducer; fixed wavelength; guided waves; interdigital electrode; piezoelectric fiber composite

\section{INTRODUCTION}

Piezoelectric fiber composites (PFC) have been utilized as smart material actuators in vibration suppression and structural health monitoring (SHM) because of excellent actuating abilities and conformability. Two types of PFC with different cross-sections were developed, such as active fiber composites (AFC) [1-3] and macro-fiber composites (MFC) [4-5]. The conventional PFC are formed with unidirectional piezoelectric fibers embedded in epoxy matrix and sandwiched between two flexible polymer sheets printed with symmetrically aligned interdigital electrodes (IDE), in which the electrode fingers are perpendicular to the piezoelectric fibers. A periodically inverted electric field is induced in the spaces between two adjacent electrode fingers during poling and actuation. The PFC can expand or contract simultaneously when a periodic voltage applies to the electrodes. However, the symmetric configuration limits PFC from being short-wavelength acoustic wave transducers. Besides, most PFC devices operate in frequency range below $100 \mathrm{kHz}$. Low frequency operation is good for vibration control, but it can not satisfy demands of SHM.
The acoustic transducers in SHM applications require large sensitivity, high temporal and spatial discrimination for defect detection. It results in a need for high frequency and directive transducers. In this work, a comparison of electric field poling in PFC induced by either symmetrically or anti-symmetrically aligned IDE is numerically carried out. The present PFC has periodic deformation when it is actuated by anti-symmetric electrodes and therefore, is abbreviated as AE-PFC. A periodic finite element approach has been used to simulate both electric fields induced by symmetric or anti-symmetric IDE as well as the interaction between AE-PFC and the host plate. The devised transducers transmit Lamb waves at frequency close to $1 \mathrm{MHz}$. The operating frequency depends on the properties of AE-PFC and the host plate. The added mass effect on the transmitted Lamb modes due to coverage of AE-PFC is studied. The transmitted waveforms were optically measured by a laser Doppler vibrometer. The near-field interference pattern shows that constant wavelength Lamb modes can be actually launched.

\section{FORMULATION}

\section{A. Configuration of $A E-P F C$}

An exploded view diagram of the AE-PFC is schematically shown in Fig. 1. Two sheets of polyimide films printed with IDE are placed on the top and bottom surfaces of unidirectional PZT-5A fibers (Advanced Cerametrics Inc., Lambertville, New Jersey) embedded in epoxy resin. Average diameter of the piezoelectric fibers is about $250 \mu \mathrm{m}$. The electrode fingers are anti-symmetrically aligned with $\lambda / 4$ offset measured from center to center to form an AE-PFC. Besides the IDE, two sets of zigzag electrodes might distribute on both top and bottom sheets. The auxiliary electrodes facilitate yielding intensive poling electric field. A piecewise inverted electric field is symmetrically generated (Fig. 2a). In contrast, the IDE alone yields an anti-symmetric electric field for excitation (Fig. 2b). The excited electric field is along or against the polarization alternately. The electrode pitch $p$ is equal to the sum of electrode width $w$ and space $s$ between two adjacent electrode fingers printed on the top and bottom polymer films. The constant pitch is one-fourth of the wavelength $\lambda, p=\lambda / 4$. 


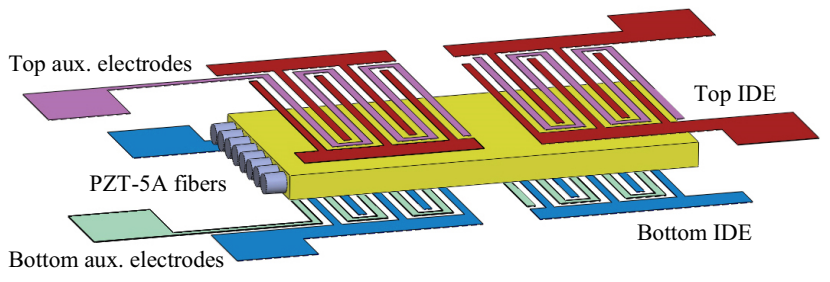

Figure 1. An exploded view diagram of AE-PFC.

(a)

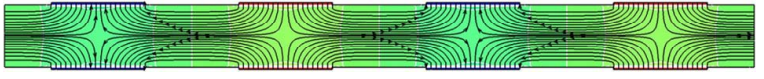

(b)

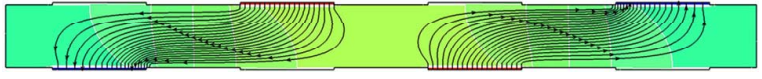

Figure 2. Electric field lines induced by (a) symmetric and (b) antisymmetric electrodes placed on the top and bottom surfaces of PFC.

\section{B. Periodic Finite Element Analysis}

A periodic finite element approach has been used to determine the electric field created in the PFC layer and timeharmonic response. The time-harmonic dependence $\exp (-i \omega t)$ is suppressed in all field quantities where $\omega$ is the angular frequency. The discretized equations of motion is of the form

$$
\left(\left[\begin{array}{ll}
\mathbf{K}_{u u} & \mathbf{K}_{u \varphi} \\
\mathbf{K}_{\varphi u} & \mathbf{K}_{\varphi \varphi}
\end{array}\right]-i \omega\left[\begin{array}{ll}
\mathbf{C} & \mathbf{0} \\
\mathbf{0} & \mathbf{0}
\end{array}\right]+\omega^{2}\left[\begin{array}{rr}
\mathbf{M} & \mathbf{0} \\
\mathbf{0} & \mathbf{0}
\end{array}\right]\right)\left\{\begin{array}{l}
\mathbf{u} \\
\boldsymbol{\varphi}
\end{array}\right\}=\left\{\begin{array}{l}
\mathbf{F} \\
\mathbf{Q}
\end{array}\right\},
$$

where $\mathbf{K}_{u u}, \mathbf{K}_{u \varphi}, \mathbf{K}_{\varphi u}, \mathbf{K}_{\varphi \varphi}, \mathbf{C}$ and $\mathbf{M}$ are the global stiffness, damping and mass matrices; $u$ and $\varphi$ are the vectors of nodal displacements and nodal electric potentials; $\mathrm{F}$ and $\mathrm{Q}$ are the vectors of nodal forces and nodal surface charges. Consider an elastic wave propagates along axial direction through a onedimensional periodic structure which comprises an infinite number of repeating cells. The degrees of freedom in each cell are equal to the corresponding dofs in the adjacent cell multiplied by a factor $\exp (i k d)$ [6]. The factor $k$ corresponds to the axial wave number, $k=2 \pi / \lambda+i \alpha$, where $\lambda$ and $\alpha$ are the wavelength and attenuation, respectively. The periodic boundary conditions are

$$
\mathbf{S}\left(x_{R}\right)=\mathbf{S}\left(x_{L}\right) e^{i k d} .
$$

where $\mathbf{S}\left(x_{R}\right)$ and $\mathbf{S}\left(x_{L}\right)$ are the vectors of nodal dofs at $\mathrm{x}_{R}$ and $x_{L}$, and $d=\mathrm{x}_{R}-x_{L}$. As an example, let the distance $d$ be equal to $\lambda$, both vectors satisfy the following relation: $\mathbf{S}\left(x_{R}\right)=\mathbf{S}\left(x_{L}\right) e^{-\alpha d}$.

The AE-PFC can be modeled by a number of repeating cells. Each cell occupies a length of four electrode pitches. It comprises a PZT-5A fiber surrounded by epoxy resin and sandwiched between two sheets of polyimide. Without loss of generality, the ANSYS finite-element code is applied to numerical simulation. The representative cell for an AE-PFC adhered to the top surface of the host plate is discretized by a finite number of elements (Fig. 3). In the representative cell, the host plate has the same depth as AE-PFC, but extends with length $L$ at both ends.

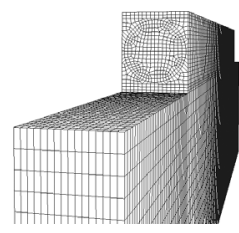

(a)

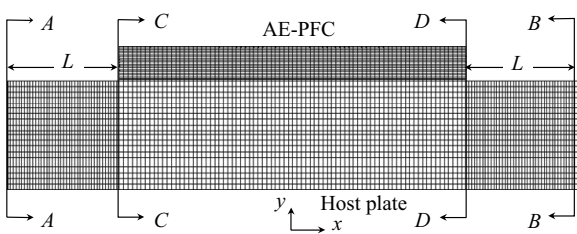

(b)
Figure 3. Finite element meshes of a representative cell: (a) end view and (b) side view.

\section{RESULTS AND DISCUSSION}

\section{A. Electric Fields}

A finite element analysis has been used to determine the steady-state electric field induced by applying static voltage of 2,000 volts to the periodic electrodes in PFC. As an example, the electrode pitch of an AE-PFC is set to be $0.8 \mathrm{~mm}$. All the corresponding dofs at both opposite surfaces in depth and ends of the AE-PFC are assumed to be equal. The piezoelectric fibers are surrounded with low permittivity epoxy resin. The electric field lines concentrate in a small region close to the diameter as they pass through the space between two adjacent fibers.

Fig. 4 shows a comparison of axial component of electric field for symmetric and anti-symmetric electrode arrangements. The results indicate that the electric field intensity induced by symmetric electrode layout is about twice as large as that of anti-symmetric arrangement. Symmetric electrode layout requires lower poling voltage than antisymmetric arrangement. The influences of electrode width and gap between adjacent electrodes on the electric field strength are demonstrated by this example. The simulation results reveal that the constant pitch IDE with larger ratio $w / s$ can achieve higher electric field.

\section{B. Interaction between AE-PFC and The Host Plate}

Refer to Fig. 3, the host plate is assumed to have the same length of AE-PFC and the periodic boundary conditions are applied to the end cross sections $\mathrm{CC}$ and DD, respectively. Let the single AE-PFC be excited by an ac sweep voltage. The minimum value of the simulated impedance curve (red line in Fig. 5) occurs at $935 \mathrm{kHz}$, which corresponds to the resonant frequency for the AE-PFC of full wavelength $3.2 \mathrm{~mm}$. The blue line indicates an impedance curve for the AE-PFC adhered to a $1 \mathrm{~mm}$ thick aluminum plate. The resonant frequency increases up to $1.03 \mathrm{MHz}$ since the stiffness of the host plate is superior to that of AE-PFC.

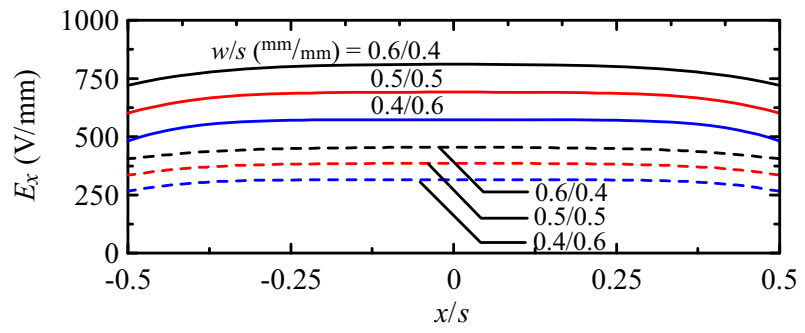

Figure 4. Comparison of electric fields $E_{x}$ induced by applying static voltage to symmetric (solid lines) and anti-symmetric electrodes (dashed lines). 
The corresponding resonant modes are illustrated by displacement contours shown in Fig. 6. In this figure and thereafter, the blue and red contours represent the displacement components toward the positive and negative directions of the $x$ - and $y$-axis, respectively. The axial displacement contours are perpendicular to the fiber orientation except in the areas close to the center and both ends. It reveals that the fundamental extensional vibration mode is yielded in the AE-PFC. According to the symmetry of deformation against the midplane, the Lamb waves propagating in a single plate are classified into symmetric and anti-symmetric modes. The neutral surface locates beyond the mid-plane of the host plate because of the AE-PFC adhered on the top surface. Therefore, both symmetric and anti-symmetric vibration with respect to the mid-plane of the host plate will be actuated by the extensional excitation of AE-PFC.

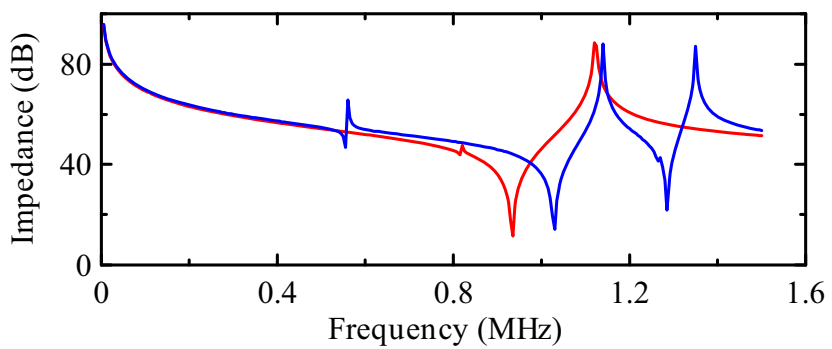

Figure 5. Simulated impedance curve of a $0.8 \mathrm{~mm}$ pitch AE-PFC (red line) and that of the AE-PFC adhered to a $1 \mathrm{~mm}$ thick aluminum plate (blue line).

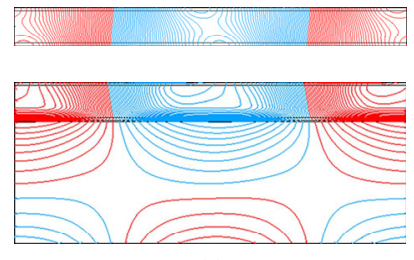

(a)

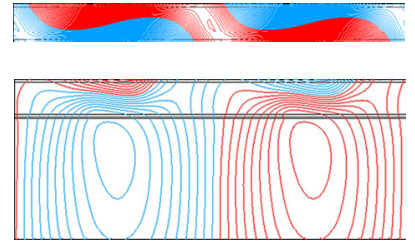

(b)
Figure 6. Contours of (a) axial and (b) transverse displacements in a fullwavelength AE-PFC excited by sinusoidal votage at $935 \mathrm{kHz}$ (top) and a 1 $\mathrm{mm}$ thick aluminum host plate actuated by the top surface adhered AE-PFC at $1.03 \mathrm{MHz}$ (bottom).

\section{Added Mass Effect}

The surface adhered AE-PFC alters the local stiffness of the host plate. The transmitted Lamb mode in the area adhered to AE-PFC has a different wavelength other than the free Lamb wave. The host plate with various extended lengths is excited by the surface adhered AE-PFC at $1.03 \mathrm{MHz}$, which is the resonant frequency of extensional mode in the abovementioned compound structure. The periodic boundary conditions are applied to both end cross sections AA and BB of the extended plate. The mechanical response attains maximum value if the extended length equals to the resonant length. The latter approximates an integer multiple of one-half the wavelength of Lamb mode. The responses at five equal interval points on the top surface of the extended regions are calculated as the indicators of resonance. Fig. 7 depicts the amplitude spectra of axial strains at those points versus the extended length. The added mass effect caused by the AE-PFC plays a non-ignorable role in resonance.

The resonant modes corresponding to different resonant lengths can be expressed by contours of displacements excited in the compound structures. Fig. 8 shows the contours of axial displacement in the host plates with various extended length. The axial displacement contours in the extended regions exhibit regularly anti-symmetric distributions with respect to the mid-plane. The displacement contours of the resonant modes are similar to each other. Numerical results indicate that the $\mathrm{A}_{0}$ mode Lamb waves are transmitted through the host plate. The resonant lengths approximate to the integer multiples of one-half wavelength of $\mathrm{A}_{0}$ mode.

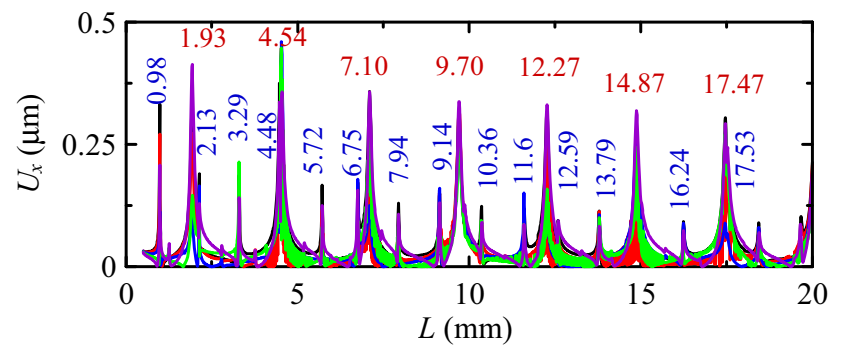

Figure 7. Spectra of calculated axial strain vs. the extended length of a $1 \mathrm{~mm}$ thick aluminum plate which is excited by a $0.8 \mathrm{~mm}$ pitch AE-PFC.

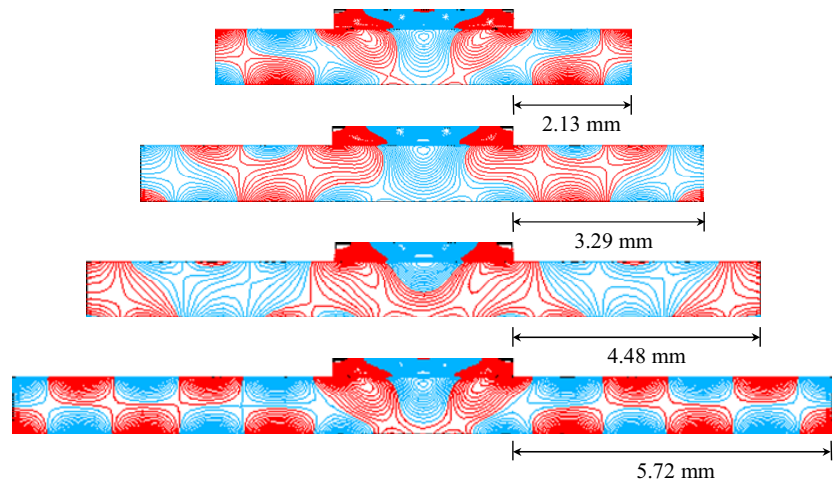

Figure 8. Contours of the excited axial displacements for the $\mathrm{A}_{0}$ Lamb mode in a representative cell corresponding to various resonant length.

On the other hands, the axial displacement contours for the $\mathrm{S}_{0}$ Lamb mode in the host plates with different extended length are shown in Fig. 9. The contours are almost perpendicular to the longitudinal axis. It is notable that no matter what kind of Lamb mode is transmitted through the host plate, the AE-PFC adhered on the top of the host plate is substantially dominated by the full-wavelength extensional mode illustrated in Fig. 6.

Fig. 10 shows the frequency spectra of Lamb waves propagating in an aluminum plate. Only two fundamental modes can be actually excited within the frequency range from $\mathrm{dc}$ to the cut-off frequency of $\mathrm{A}_{1}$ mode, approximately 1.6 $\mathrm{MHz}$. The wavelength for $\mathrm{A}_{0}$ and $\mathrm{S}_{0}$ mode Lamb waves at frequency $1.03 \mathrm{MHz}$ (marked by a dashed line) are 2.276 and $5.148 \mathrm{~mm}$. Fig. 11 depicts the deviation between the resonant length and the integer multiple of one-half wavelength of Lamb mode decreases as the extended length increases. The added 
mass effect of PFC has more notable influence on transmission of $\mathrm{S}_{0}$ modes than $\mathrm{A}_{0}$ mode in the area near both edges of PFC.

\section{Near-field Interference Pattern}

The transmitted acoustic waveforms were measured along the axis in front of a prototype AE-PFC adhered to a $30 \mathrm{~mm}$ wide, $60 \mathrm{~mm}$ long, and $1 \mathrm{~mm}$ thick aluminum plate by a laser Doppler vibrometer. The prototype AE-PFC has an aperture of $30 \mathrm{~mm}$ and center frequency of $780 \mathrm{kHz}$. The linear scan results (Fig. 12) reveal the near-field interference pattern. The amplitudes of measured waveforms are oscillating. It is usually found in near-field of bulk wave ultrasonic transducers with large aperture. The experimental results indicate that the AEPFC can transmit guided waves of constant wavelength.

\section{CONCLUSION}

A periodic finite-element approach was used to model the $\mathrm{A}_{0}$ and $\mathrm{S}_{0}$ Lamb waves actuated by a novel AE-PFC ultrasonic transducer with center frequency of near $1 \mathrm{MHz}$. The flexible AE-PFC transducer can be poled by either symmetric or antisymmetric interdigital electrodes, but must be actuated by antisymmetric electrodes. Numerical simulation confirms the extensional vibration mode excited in AE-PFC is viable to transmit fixed-wavelength structural guided waves for potential applications in structural health monitoring. The auxiliary zigzag electrodes on the top and bottom sheets can assist yielding intensive poling electric field. The added mass effect of PFC has more notable influence on transmission of $\mathrm{S}_{0}$ modes than $\mathrm{A}_{0}$ mode in the area near both edges of PFC. The linear scanning results of transmitted acoustic waveforms along the axis in front of a wide aperture AE-PFC transducer reveals the near-field acoustic interference pattern. It provides sufficient evidence that the present AE-PFC can transmit guided waves of constant wavelength.

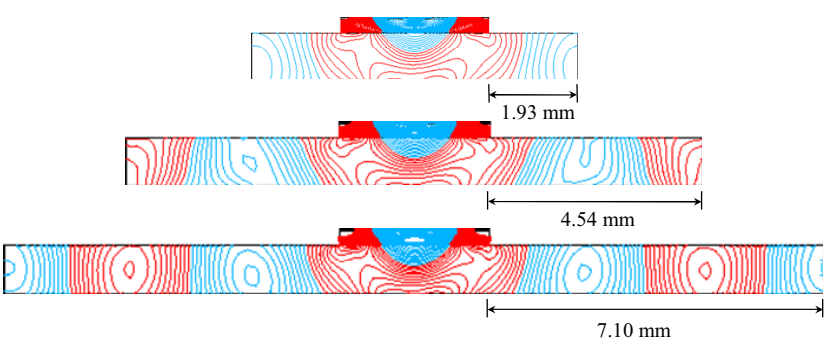

Figure 9. Contours of the excited axial displacements for the $\mathrm{S}_{0}$ Lamb mode in a representative cell corresponding to different resonant length.

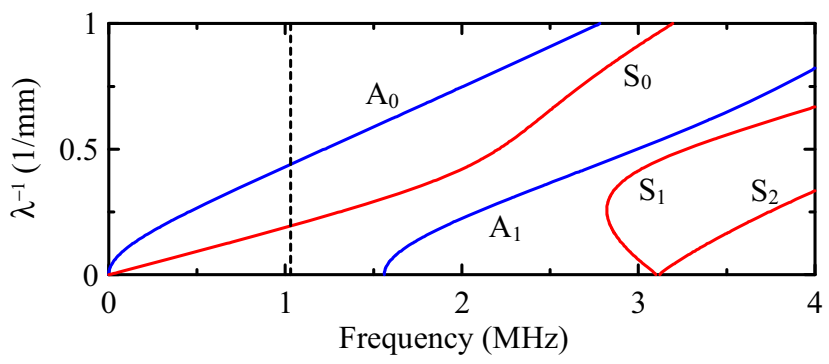

Figure 10. Frequency spectra of Lamb modes in a $1 \mathrm{~mm}$ thick aluminum plate.

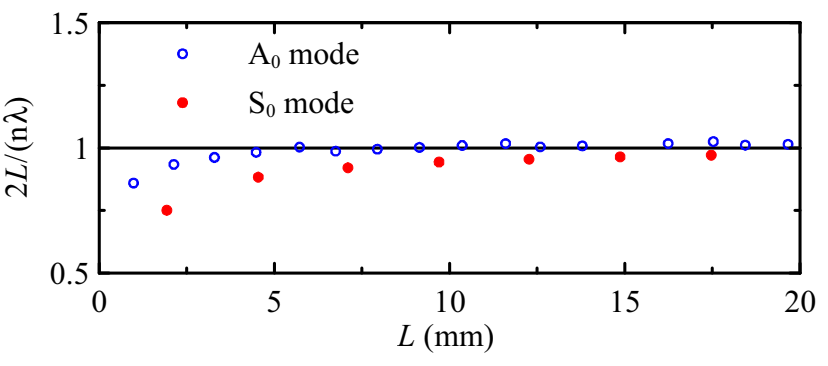

Figure 11. The ratio of resonant length to the multiple of one-half the wavelength of Lamb mode with respect to the resonant length.

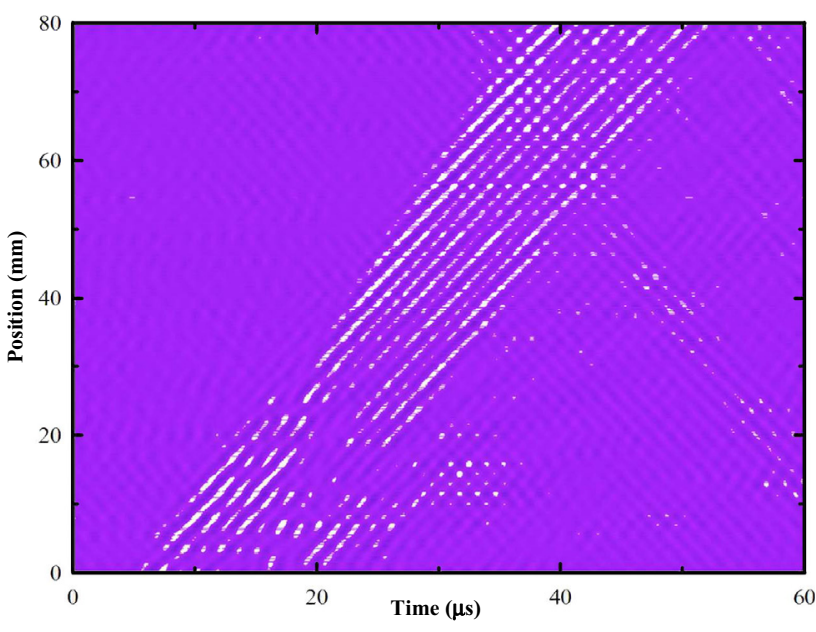

Figure 12. Amplitude of Lamb modes measured along the axial direction in front of a $780 \mathrm{kHz}$ AE-PFC with $30 \mathrm{~mm}$ aperture.

\section{ACKNOWLEDGMENT}

This work was supported by the National Science Council of the Republic of China through the grant NSC101-2221-E009-021-MY2.

\section{REFERENCES}

[1] A.A. Bent and N.W. Hagood, "Piezoelectric fiber composites with interdigitated electrodes," J. Intel. Mat. Syst. Str. vol. 6, pp. 338-349, 1997.

[2] A.J. Brunner, M. Barbezat, C. Huber, and P.H. Flüeler, "The potential of active fiber composites made from piezoelectric fibers for actuating and sensing applications in structural health monitoring," Mater. Struct., vol. 38 , pp. 561-567, 2005.

[3] M. Barbezat, A.J. Brunner, C. Huber, and P.H. Flüeler, "Integrated active fiber composite elements: characterization for acoustic emission and acousto-ultrasonics," J. Intel. Mat. Syst. Str., vol. 18, pp. 515-525, 2007.

[4] W.K. Wilkie, R.G. Bryant, J.W. High, R.L. Fox, R.F. Hellbaum, A Jalink, B.D. Little Jr., P.H. Mirick, "Low-cost piezocomposite actuator for structural control applications," Proc. SPIE 3991, Smart Structures and Materials, pp. 323-334, 2000.

[5] M.S. Azzouz, C. Mei, J.S. Bevan, and J.J. Ro, "Finite element modeling of MFC/AFC actuators and performance of MFC," J. Intel. Mat. Syst. Str., vol. 12, pp. 601-612, 2001.

[6] D.J. Mead, "A general theory of harmonic wave propagation in linear periodic systems with multiple coupling," J. Sound Vib., vol. 27, pp 235-260, 1973. 